\title{
POSITIVE SOLUTION OF A FRACTIONAL DIFFERENTIAL EQUATION WITH INTEGRAL BOUNDARY CONDITIONS
}

\author{
Mohammed S. Abdo ${ }^{1,2}$, Hanan A. Wahash ${ }^{1}$, Satish K. Panchal ${ }^{3}$ \\ ${ }^{1}$ Research Scholar at Department of Mathematics, Dr. Babasaheb Ambedkar Marathwada University \\ Aurangabad 431004 (M.S.), India \\ ${ }^{2}$ Department of Mathematics, Hodeidah University \\ Al-Hodeidah, Yemen \\ ${ }^{3}$ Department of Mathematics, Dr. Babasaheb Ambedkar Marathwada University \\ Aurangabad 431004 (M.S.), India \\ msabdo1977@gmail.com,drpanchalskk@gmail.com
}

Received: 15 May 2018; Accepted: 8 November 2018

\begin{abstract}
In this paper, we prove the existence and uniqueness of a positive solution for a boundary value problem of nonlinear fractional differential equations involving a Caputo fractional operator with integral boundary conditions. The technique used to prove our results depends on the upper and lower solution, the Schauder fixed point theorem and the Banach contraction principle. The result of existence obtained through constructing the upper and lower control functions of the nonlinear term without any monotone requirement. Illustrative examples are provided.
\end{abstract}

MSC 2010: $34 A 08,34 B 15,34 B 18,47 H 10$

Keywords: fractional derivative and integral, positive solutions of nonlinear boundary value problems, upper and lower solutions, fixed point theorem

\section{Introduction}

In recent years, the theory of fractional differential equations has attracted considerable interest in mathematics and many applications such as physics, mechanics, chemistry, engineering, etc. For more details, see the monographs of Hilfer [1], Kilbas et al. [2], Miller and Ross [3], Podlubny [4] and Samko et al. [5]. Many interesting results of the existence of positive solutions of various classes of fractional differential equations with or without the integral boundary condition have been discussed. Among these works (see [6-17]) and the references therein. For example in [12], Nan and Wang studied the existence and uniqueness of a positive solution for the following nonlinear fractional differential equations

$$
\begin{aligned}
& D_{0^{+}}^{\alpha} u(t)=f(t, u(t)), t \in[0,1] \\
& u(0)=0,
\end{aligned}
$$


where $0<\alpha<1, D_{0^{+}}^{\alpha}$ is the standard Riemann-Liouville fractional derivative and $f:[0,1] \times \mathbb{R}^{+} \longrightarrow \mathbb{R}^{+}$is continuous. The authors used the method of upper and lower solutions and Schauder fixed point theorem to prove their results without any monotone requirement on the nonlinear term. Wang et al. in [14], discussed the existence of solutions of the following fractional differential equations with integral boundary conditions

$$
\begin{aligned}
& D^{\alpha} u(t)=f(t, u(t)), \quad t \in[0, T], \\
& u(0)=\lambda \int_{0}^{T} u(s) d s+d,
\end{aligned}
$$

where $0<\alpha<1, \lambda \geq 0, D^{\alpha}$ is the standard Riemann-Liouville fractional derivative and $f:[0, T] \times \mathbb{R} \longrightarrow \mathbb{R}$ is continuous. The authors applied the upper and lower solutions combined with a monotone iterative technique to obtain their results.

Nanware and Dhaigude in [18], obtained the existence and uniqueness of solution of the problem (3)-(4) by monotone iterative method without locally Holder continuity. Motivated by the above works, and inspired [12], in this paper, we used the upper and lower solution method, Schauder's fixed point theorem and Banach contraction principle to obtain the existence and uniqueness of a positive solution for the following fractional differential equations with integral boundary conditions

$$
\begin{aligned}
& { }^{c} D_{0^{+}}^{\alpha} u(t)=f(t, u(t)), t \in[0,1], \\
& u(0)=\lambda \int_{0}^{1} u(s) d s+d,
\end{aligned}
$$

where $0<\alpha<1, \lambda \geq 0, d \in \mathbb{R}^{+},{ }^{c} D_{0^{+}}^{\alpha}$ is the standard Caputo fractional operator and $f:[0,1] \times \mathbb{R}^{+} \longrightarrow \mathbb{R}^{+}$is continuous.

The paper is organized into five sections. In Section 2, the concepts of Caputo, Riemann-Liouville types with its properties and fixed point theorems are presented. In Section 3, the existence and uniqueness of positive solution to (5)-(6) are proved. In Section 4, the examples illustrative are provided. Finally, the conclusion is given in Section 5.

\section{Preliminaries}

In this section, we recall some basic definitions and necessary lemmas related to fractional calculus and fixed point theorems that will be used throughout this paper.

Let $C[0,1]$ be the Banach space endowed with the infinity norm and $K$ a nonempty closed subset of $C[0,1]$ defined as

$$
K=\{u(t) \in C[0,1]: u(t) \geq 0,0 \leq t \leq 1\} .
$$


$C^{n}[0,1]$ denotes the class of all real valued functions defined on $[0,1]$ which have a continuous $n$th order derivative.

Definition 1. [2]. The left sided Riemann-Liouville fractional integral of order $\alpha>0$ of a function $g \in C[0,1]$ is given by

$$
I_{0^{+}}^{\alpha} g(t)=\frac{1}{\Gamma(\alpha)} \int_{0}^{t}(t-s)^{\alpha-1} g(s) d s, t>0,
$$

where $\Gamma$ denotes the Gamma function.

Definition 2. [2]. Let $n-1<\alpha<n$. The left sided Riemann-Liouville fractional derivative of order $\alpha$ of a function $g:[0,1] \rightarrow \mathbb{R}$ defined by

$$
D_{0^{+}}^{\alpha} g(t)=\frac{d^{n}}{d t^{n}} I_{0^{+}}^{n-\alpha} g(t), t>0
$$

provided the right side integral is pointwise defined on $[0,1]$ and $n=[\alpha]+1$, $[\alpha]$ denotes the integer part of the real number $\alpha$.

In particular, If $0<\alpha<1$, then $D_{0^{+}}^{\alpha} g(t)=\frac{d}{d t} I_{0^{+}}^{1-\alpha} g(t), \quad t>0$.

Definition 3. [2]. Let $n-1<\alpha<n$. The left sided Caputo derivative of order $\alpha$ of a function $g \in C^{n}[0,1]$ is given by

$$
{ }^{c} D_{0^{+}}^{\alpha} g(t)=I_{0^{+}}^{n-\alpha} \frac{d^{n}}{d t^{n}} g(t), t>0 .
$$

In particular, If $0<\alpha<1$ then

$$
{ }^{c} D_{0^{+}}^{\alpha} g(t)=I_{0^{+}}^{1-\alpha} \frac{d}{d t} g(t), t>0 .
$$

Moreover, Caputo's derivative of a constant is equal to zero.

Lemma 1. [19]. Let $g \in C^{n}[0,1]$ and $\alpha>0$. Then

1. ${ }^{c} D_{0^{+}}^{\alpha} I_{0^{+}}^{\alpha} g(t)=g(t)$.

2. $I_{0^{+}}^{\alpha}{ }^{c} D_{0^{+}}^{\alpha} g(t)=g(t)-\sum_{k=0}^{n-1} \frac{g^{(k)}\left(0^{+}\right)}{k !} t^{k}$. In particular, if $0<\alpha<1$ we have

$$
I_{0^{+}}^{\alpha c} D_{0^{+}}^{\alpha} g(t)=g(t)-g(0) .
$$

Theorem 1. [20]. (Banach contraction principle). Let $(X, d)$ be a non-empty complete metric space with a contraction mapping $P: X \rightarrow X$. Then, $P$ has a unique fixed-point $x$ in $X$ (i.e. $P x=x$ ). 
Theorem 2. [20]. (Schauder fixed point theorem). Let $X$ be a Banach space and let $A$ a closed convex, bounded subset of $X$. If $P: A \longrightarrow A$ is a continuous map such that the set $\{P x: x \in A\}$ is relatively compact in $X$. Then $P$ has at least one fixed point.

Definition 4. A function $u \in C[0,1] \cap C^{1}[0,1]$ is said to be a solution of (5)-(6) if $u$ satisfies the equation ${ }^{c} D_{0^{+}}^{\alpha} u(t)=f(t, u(t)), t \in[0,1]$, with integral boundary conditions $u(0)=\lambda \int_{0}^{1} u(s) d s+d$.

Definition 5. A function $u \in C[0,1] \cap C^{1}[0,1]$ is called a positive solution of the problem (5)-(6) if $u(t) \geq 0$ for all $t \in[0,1]$ and $u$ satisfies the problem (5)-(6).

Definition 6. Let $a, b \in \mathbb{R}^{+}$, and $b>a$. For any $u \in[a, b]$, we define the upper-control function $H(t, u)=\sup _{a \leq \eta \leq u} f(t, \eta)$, and lower-control function $h(t, u)=\inf _{u \leq \eta \leq b} f(t, \eta)$.

Obviously, $H(t, u)$ and $h(t, u)$ are monotonous non-decreasing on $u$ and

$$
h(t, u) \leq f(t, u) \leq H(t, u) .
$$

Definition 7. Let $\widetilde{u}(t), \widehat{u}(t) \in K$ and $a \leq \widehat{u}(t) \leq \widetilde{u}(t) \leq b$ satisfy

$$
\begin{aligned}
& { }^{c} D_{0^{+}}^{\alpha} \widetilde{u}(t) \geq H(t, \widetilde{u}(t)), \quad t \in[0,1], \\
& \widetilde{u}(0) \geq \lambda \int_{0}^{1} \widetilde{u}(s) d s+d,
\end{aligned}
$$

or

$$
\widetilde{u}(t) \geq \frac{1}{\Gamma(\alpha)} \int_{0}^{t}(t-s)^{\alpha-1} H(t, \widetilde{u}(t)) d s+\lambda \int_{0}^{1} \widetilde{u}(s) d s+d, \quad t \in[0,1]
$$

and

$$
\begin{aligned}
& { }^{c} D_{0^{+}}^{\alpha} \widehat{u}(t) \leq h(t, \widehat{u}(t)), \quad t \in[0,1] \\
& \widehat{u}(0) \leq \lambda \int_{0}^{1} \widehat{u}(s) d s+d
\end{aligned}
$$

or

$$
\widehat{u}(t) \leq \frac{1}{\Gamma(\alpha)} \int_{0}^{t}(t-s)^{\alpha-1} h(t, \widehat{u}(t)) d s+\lambda \int_{0}^{1} \widehat{u}(s) d s+d, t \in[0,1]
$$

Then the functions $\widetilde{u}(t)$ and $\widehat{u}(t)$ are called upper and lower solutions, respectively for problem (5)-(6). 


\section{Main results}

In this section, we shall give existence and uniqueness results of (5)-(6) and prove it. Before starting and proving the main results, we introduce the following lemma:

Lemma 2. Assume that $f(t, u(t)) \in C\left([0,1], \mathbb{R}^{+}\right)$, then $u(t) \in C[0,1] \cap C^{1}[0,1]$ is a solution of the boundary value problem (5)-(6) if and only if $u(t)$ is a solution of the integral equation

$$
u(t)=\frac{1}{\Gamma(\alpha)} \int_{0}^{t}(t-s)^{\alpha-1} f(s, u(s)) d s+\lambda \int_{0}^{1} u(s) d s+d, \quad t \in[0,1]
$$

Proof. Suppose $u(t)$ satisfies the problem (5)-(6), then applying $I_{0^{+}}^{\alpha}$ to both sides of Eq. (5), we have

$$
I_{0^{+}}^{\alpha}{ }^{c} D_{0^{+}}^{\alpha} u(t)=I_{0^{+}}^{\alpha} f(t, u(t)) .
$$

In view of Lemma 1 and the integral boundary condition Eq. (6), we get

$$
u(t)=\frac{1}{\Gamma(\alpha)} \int_{0}^{t}(t-s)^{\alpha-1} f(s, u(s)) d s+\lambda \int_{0}^{1} u(s) d s+d, t \in[0,1] .
$$

Conversely, suppose $u(t)$ satisfies Eq. (9). By Definition 3 and Lemma 1, then for $t \in[0,1]$, we observe that

$$
\begin{aligned}
{ }^{c} D_{0^{+}}^{\alpha} u(t) & ={ }^{c} D_{0^{+}}^{\alpha}\left(\frac{1}{\Gamma(\alpha)} \int_{0}^{t}(t-s)^{\alpha-1} f(s, u(s)) d s+\lambda \int_{0}^{1} u(s) d s+d\right) \\
& ={ }^{c} D_{0^{+}}^{\alpha} I_{0^{+}}^{\alpha} f(t, u(t))+{ }^{c} D_{0^{+}}^{\alpha}\left(\lambda \int_{0}^{1} u(s) d s+d\right) \\
& =f(t, u(t)) .
\end{aligned}
$$

Moreover, the integral boundary condition $u(0)=\lambda \int_{0}^{1} u(s) d s+d$ holds.

The first result shows that $P: K \rightarrow K$ is compact. Here, consider the operator $P: K \rightarrow K$ defined by

$$
P u(t)=\frac{1}{\Gamma(\alpha)} \int_{0}^{t}(t-s)^{\alpha-1} f(s, u(s)) d s+\lambda \int_{0}^{1} u(s) d s+d, \quad t \in[0,1] .
$$

Lemma 3. Assume that $f \in C\left([0,1], \mathbb{R}^{+}\right)$. Then the mapping $P: K \rightarrow K$ is compact. $\square$ ProOF. The operator $P: K \rightarrow K$ is continuous in view of the hypothesis of nonnegativeness and continuity of $f(t, u)$.

Let $\mathbb{S} \subset K$ be bounded, which is to say there exists a constant $M>0$ such that $\|u\| \leq M$ for all $u \in \mathbb{S}$, and let the function $f:[0,1] \times \mathbb{S} \rightarrow \mathbb{R}$ be bounded by $L$ i.e.

$$
L=\max _{(t, u) \in[0,1] \times[0, M]} f(t, u(t))+1 .
$$


From Eq. (10), then for any $u \in \mathbb{S}$ and for each $t \in[0,1]$, we have

$$
\begin{aligned}
|(P u)(t)| & =\left|\frac{1}{\Gamma(\alpha)} \int_{0}^{t}(t-s)^{\alpha-1} f(s, u(s)) d s+\lambda \int_{0}^{1} u(s) d s+d\right| \\
& \leq \frac{1}{\Gamma(\alpha)} \int_{0}^{t}(t-s)^{\alpha-1}|f(s, u(s))| d s+\lambda \int_{0}^{1}|u(s)| d s+d \\
& \leq \frac{L t^{\alpha}}{\Gamma(\alpha+1)}+\lambda M+d
\end{aligned}
$$

Thus,

$$
\|P u\| \leq \frac{L}{\Gamma(\alpha+1)}+\lambda M+d .
$$

Hence, $P(\mathbb{S})$ is uniformly bounded.

Now, we will prove that the operator $P$ is equicontinuous. For each $u \in \mathbb{S}$. Then for $t_{1}, t_{2} \in[0,1]$ with $t_{1}<t_{2}$, we have

$$
\begin{aligned}
& \left|(P u)\left(t_{1}\right)-(P u)\left(t_{2}\right)\right| \\
& =\left|\frac{1}{\Gamma(\alpha)} \int_{0}^{t_{1}}\left(t_{1}-s\right)^{\alpha-1} f(s, u(s)) d s-\frac{1}{\Gamma(\alpha)} \int_{0}^{t_{2}}\left(t_{2}-s\right)^{\alpha-1} f(s, u(s)) d s\right| \\
& =\mid \frac{1}{\Gamma(\alpha)} \int_{0}^{t_{1}}\left(t_{1}-s\right)^{\alpha-1} f(s, u(s)) d s-\frac{1}{\Gamma(\alpha)} \int_{0}^{t_{1}}\left(t_{2}-s\right)^{\alpha-1} f(s, u(s)) d s \\
& +\frac{1}{\Gamma(\alpha)} \int_{0}^{t_{1}}\left(t_{2}-s\right)^{\alpha-1} f(s, u(s)) d s-\frac{1}{\Gamma(\alpha)} \int_{0}^{t_{2}}\left(t_{2}-s\right)^{\alpha-1} f(s, u(s)) d s \mid \\
& =\mid \frac{1}{\Gamma(\alpha)} \int_{0}^{t_{1}}\left(t_{1}-s\right)^{\alpha-1} f(s, u(s)) d s-\frac{1}{\Gamma(\alpha)} \int_{0}^{t_{1}}\left(t_{2}-s\right)^{\alpha-1} f(s, u(s)) d s \\
& -\frac{1}{\Gamma(\alpha)} \int_{t_{1}}^{t_{2}}\left(t_{2}-s\right)^{\alpha-1} f(s, u(s)) d s \mid \\
& \leq \frac{1}{\Gamma(\alpha)} \int_{0}^{t_{1}}\left|\left(t_{1}-s\right)^{\alpha-1}-\left(t_{2}-s\right)^{\alpha-1}\right||f(s, u(s))| d s \\
& +\frac{1}{\Gamma(\alpha)} \int_{t_{1}}^{t_{2}}\left(t_{2}-s\right)^{\alpha-1}|f(s, u(s))| d s \\
& \leq \frac{L}{\Gamma(\alpha)} \int_{0}^{t_{1}}\left(\left(t_{1}-s\right)^{\alpha-1}-\left(t_{2}-s\right)^{\alpha-1}\right) d s+\frac{L}{\Gamma(\alpha)} \int_{t_{1}}^{t_{2}}\left(t_{2}-s\right)^{\alpha-1} d s \\
& \leq \frac{L}{\Gamma(\alpha+1)}\left(t_{1}^{\alpha}+\left(t_{2}-t_{1}\right)^{\alpha}-t_{2}^{\alpha}+\left(t_{2}-t_{1}\right)^{\alpha}\right) \\
& \leq \frac{2 L}{\Gamma(\alpha+1)}\left(t_{2}-t_{1}\right)^{\alpha} \text {. }
\end{aligned}
$$

As $t_{1} \rightarrow t_{2}$, the right-hand side of the inequality Eq. (11) tends to zero and the convergence is independent of $u$ in $\mathbb{S}$, which means that $P(\mathbb{S})$ is equicontinuous. Thus, the compactness of $P$ follows by Ascoli Arzela's theorem. 
The second result proves an existence of the solution of problem (5)-(6) by means of the Schauder fixed point theorem.

Theorem 3. Assume that $f:[0,1] \times \mathbb{R}^{+} \rightarrow \mathbb{R}^{+}$is continuous, and $\widetilde{u}(t), \widehat{u}(t)$ are upper and lower solutions of problem (5)-(6). Then there exists at least a solution $u(t)$ of the integral boundary value problem (5)-(6). Moreover,

$$
\widehat{u}(t) \leq u(t) \leq \widetilde{u}(t), \quad t \in[0,1] .
$$

ProOF. Define the set

$$
\Lambda=\{v(t): v(t) \in K, \widehat{u}(t) \leq v(t) \leq \widetilde{u}(t), t \in[0,1]\},
$$

endowed with norm $\|v(t)\|=\max _{t \in[0,1]}|v(t)| \leq b$. Hence $\Lambda$ is a convex, closed and bounded subset of the Banach space $C\left([0,1], \mathbb{R}^{+}\right)$. According to Lemma 3 , the operator $P: K \rightarrow K$ is compact. To apply Schauder's fixed point theorem, we need only to prove $P: \Lambda \rightarrow \Lambda$. Indeed, for any $v(t) \in \Lambda$, then $\widehat{u}(t) \leq v(t) \leq \widetilde{u}(t)$ and by the Definitions 6, 7, we have

$$
\begin{aligned}
P v(t) & =\frac{1}{\Gamma(\alpha)} \int_{0}^{t}(t-s)^{\alpha-1} f(s, v(s)) d s+\lambda \int_{0}^{1} v(s) d s+d \\
& \leq \frac{1}{\Gamma(\alpha)} \int_{0}^{t}(t-s)^{\alpha-1} H(s, v(s)) d s+\lambda \int_{0}^{1} v(s) d s+d \\
& \leq \frac{1}{\Gamma(\alpha)} \int_{0}^{t}(t-s)^{\alpha-1} H(s, \widetilde{u}(s)) d s+\lambda \int_{0}^{1} \widetilde{u}(s) d s+d \\
& \leq \widetilde{u}(t)
\end{aligned}
$$

and

$$
\begin{aligned}
P v(t) & =\frac{1}{\Gamma(\alpha)} \int_{0}^{t}(t-s)^{\alpha-1} f(s, v(s)) d s+\lambda \int_{0}^{1} v(s) d s+d \\
& \geq \frac{1}{\Gamma(\alpha)} \int_{0}^{t}(t-s)^{\alpha-1} h(s, v(s)) d s+\lambda \int_{0}^{1} v(s) d s+d \\
& \geq \frac{1}{\Gamma(\alpha)} \int_{0}^{t}(t-s)^{\alpha-1} h(s, \widehat{u}(s)) d s+\lambda \int_{0}^{1} \widehat{u}(s) d s+d \\
& \geq \widehat{u}(t) .
\end{aligned}
$$

Therefore, $\widehat{u}(t) \leq P v(t) \leq \widetilde{u}(t), 0 \leq t \leq 1$ which implies that $P v(t) \in \Lambda$ for all $t \in[0,1]$. This proves that $P: \Lambda \rightarrow \Lambda$.

As consequence of Theorem 2, the operator $P$ has at least one fixed point $u(t) \in \Lambda$, $0 \leq t \leq 1$. Therefore, the integral boundary value problem (5)-(6) has at least one solution $u(t) \in C[0,1]$ and $\widetilde{u}(t) \geq u(t) \geq \widehat{u}(t), t \in[0,1]$.

The final result is based on the Banach contraction principle. 
Theorem 4. Assume that $f:[0,1] \times \mathbb{R}^{+} \rightarrow \mathbb{R}^{+}$is continuous and there exists a constant $\ell>0$ such that

$$
\begin{aligned}
& \|f(t, u)-f(t, v)\| \leq \ell\|u-v\|, t \in[0,1], u, v \in C[0,1] \text {. } \\
& \text { If }\left(\frac{\ell}{\Gamma(\alpha+1)}+\lambda\right)<1 \text {. Then the problem (5)-(6) has a unique solution } \\
& u(t) \in C[0,1] \text {. }
\end{aligned}
$$

PROOF. Theorem 3 shows that the problem (5)-(6) has at least one positive solution in $\Lambda$ given by

$$
u(t)=\frac{1}{\Gamma(\alpha)} \int_{0}^{t}(t-s)^{\alpha-1} f(s, u(s)) d s+\lambda \int_{0}^{1} u(s) d s+d, t \in[0,1] .
$$

Hence, we need only to prove that the operator $P$ defined by

$$
(P u)(t)=\frac{1}{\Gamma(\alpha)} \int_{0}^{t}(t-s)^{\alpha-1} f(s, u(s)) d s+\lambda \int_{0}^{1} u(s) d s+d, \quad t \in[0,1]
$$

is contract in $C[0,1]$. Indeed, by Eq. (12), then for $u_{1}, u_{2} \in C[0,1]$ and $t \in[0,1]$, we have

$$
\begin{aligned}
& \left\|\left(P u_{1}\right)(t)-\left(P u_{2}\right)(t)\right\| \\
\leq & \frac{1}{\Gamma(\alpha)} \int_{0}^{t}(t-s)^{\alpha-1}\left\|f\left(s, u_{1}(s)\right)-f\left(s, u_{2}(s)\right)\right\| d s+\lambda \int_{0}^{1}\left\|u_{1}(s)-u_{2}(s)\right\| d s \\
\leq & \frac{\ell}{\Gamma(\alpha)} \int_{0}^{t}(t-s)^{\alpha-1}\left\|u_{1}(s)-u_{2}(s)\right\|+\lambda \int_{0}^{1}\left\|u_{1}(s)-u_{2}(s)\right\| d s \\
\leq & \left(\frac{\ell}{\Gamma(\alpha+1)}+\lambda\right)\left\|u_{1}(t)-u_{2}(t)\right\| .
\end{aligned}
$$

Since $\left(\frac{\ell}{\Gamma(\alpha+1)}+\lambda\right)<1$, then $P$ is contraction mapping. As consequence of Theorem 1, we can conclude that $P$ has a unique fixed point which is the unique solution of (5)-(6) on $[0,1]$.

\section{Examples}

In this section, we give two examples to illuminate our results.

Example 1. Consider the fractional differential equation with integral boundary condition

$$
{ }^{c} D_{0^{+}}^{\frac{1}{2}} u(t)=1+\frac{u(t)}{(1+\sin (u(t)))}, t \in[0,1]
$$




$$
u(0)=\frac{2}{3} \int_{0}^{1} u(s) d s+\frac{1}{2}
$$

For $0<u, v<+\infty$, and for $t \in[0,1]$, we have

$$
\|f(t, u(t))-f(t, v(t))\| \leq\left\|\frac{u(t)-v(t)}{(1+\sin (u(t)))(1+\sin (v(t)))}\right\| \leq \frac{1}{4}\|u(t)-v(t)\| .
$$

Therefore, the condition $\left(\frac{\ell}{\Gamma(\alpha+1)}+\lambda\right)<1$ holds with $\alpha=\frac{1}{2}, \ell=\frac{1}{4}$ and $\lambda=\frac{2}{3}$. Indeed, $\left(\frac{\frac{1}{4}}{\Gamma\left(\frac{1}{2}+1\right)}+\frac{2}{3}\right) \simeq 0.9<1$. By Theorem 1 , the fractional differential eqaution (13)-(14) has the unique positive solution $u(t) \in C[0,1]$.

Example 2. Consider the fractional differential equation with integral boundary condition

$$
\begin{aligned}
& { }^{c} D_{0^{+}}^{\frac{1}{3}} u(t)=\frac{1}{2} \sin t, t \in\left[\frac{1}{2}, 1\right], \\
& u(0)=\frac{1}{4} \int_{\frac{1}{2}}^{1} u(s) d s,
\end{aligned}
$$

where $\lambda=\frac{1}{4}$ and $d=0$.

Using the Laplace transform of the Caputo fractional derivative, we get

$$
u(t)=\frac{1}{2} t^{\frac{3}{2}} E_{2, \frac{5}{2}}\left(-t^{2}\right)+0.063675 \overline{3}
$$

where $E_{\alpha, \beta}(z)=\sum_{k=0}^{\infty} \frac{z^{k}}{\Gamma(\alpha k+\beta)}, z \in \mathbb{C}$, is called the Mittage-Leffler function.

It is clear via Theorem 4 and Theorem 3 that the above problem (15)-(16) has a unique positive solution $u(t) \in C\left[\frac{1}{2}, 1\right], t \in\left[\frac{1}{2}, 1\right]$. Moreover, $\widehat{u}(t) \leq u(t) \leq \widetilde{u}(t)$ where

$$
\widetilde{u}(t)=\frac{1}{2} t^{\frac{3}{2}} E_{2, \frac{5}{2}}\left(-t^{2}\right)+1 \text { and } \widehat{u}(t)=\frac{1}{2} t^{\frac{3}{2}} E_{2, \frac{5}{2}}\left(-t^{2}\right)
$$

are respectively the upper and lower solutions of the problem (15)-(16).

\section{Conclusions}

We can conclude that the main results of this article have been successfully achieved, that is, through the Banach fixed point theorem and the Schauder fixed point theorem, extremely important results within the mathematical analysis, we scrutinized the existence and uniqueness of positive solution of the Integral boundary value 
problem for nonlinear fractional differential equation introduced by the Caputo fractional derivative. The result of existence obtained through the constructing the upper and lower control functions of the nonlinear term without any monotone requirement as opposed to what has already been studied by Wang et al. in [14] and Nanware et al. in [18]. This paper contributes to the growth of fractional calculus, especially in the case of fractional differential equations involving a Caputo fractional derivative with integral boundary conditions. There are some articles that carried out a brief study on the existence and uniqueness of solutions of fractional differential equations, and one of the objectives of this paper is to contribute so that it can have a greater extent of studies within the mathematical analysis related to fractional differential equations.

\section{Acknowledgment}

The authors are grateful to the referees for the careful reading of the paper and for their remarks.

\section{References}

[1] Hilfer, R. (2000). Applications of Fractional Calculus in Physics. Singapore: World Scientific Publ. Co.

[2] Kilbas, A.A., Srivastava, H.M., \& Trujillo, J.J. (2006). Theory and Applications of Fractional Differential Equations. North-Holland Math. Stud., 204, Amsterdam: Elsevier.

[3] Miller, K.S.,\& Ross, B. (1993). An Introduction to the Fractional Calculus and Differential Equations. New York: John Wiley.

[4] Podlubny, I. (1999). Fractional Differential Equations. San Diego: Academic Press.

[5] Samko, S.G., Kilbas, A.A., \& Marichev, O.I. (1993). Fractional Integrals and Derivatives. Theory and Applications. Yverdon: Gordon and Breach.

[6] Abdo, M.S., \& Panchal, S.K. (2018). Effect of perturbation in the solution of fractional neutral functional differential equations. Journal of the Korean Society for Industrial and Applied Mathematics, 22(1), 63-74.

[7] Abdo, M.S., \& Panchal, S.K. (2017). Existence and continuous dependence for fractional neutral functional differential equations. Journal Mathematical Modeling, 5(2), 153-170.

[8] Abdo, M.S., \& Panchal, S.K. (2018). Some new uniqueness results of solutions to nonlinear fractional integro-differential equations. Annals of Pure and Applied Mathematics, 16(2), 345-352.

[9] Abdo, M.S., \& Panchal, S.K. (2018). Weighted fractional neutral functional differential equations. J. Sib. Fed. Univ. Math. Phys., 11(5), 535-549.

[10] Cabada, A., \& Wang, G. (2012). Positive solutions of nonlinear fractional differential equations with integral boundary value conditions. Journal of Mathematical Analysis and Applications, 389(1), 403-411.

[11] Chidouh, A., Guezane-Lakoud, A., \& Bebbouchi, R. (2016). Positive solutions of the fractional relaxation equation using lower and upper solutions. Vietnam Journal of Mathematics, 44(4), 739-748.

[12] Nan, L., \& Changyou, W. (2013). New existence results of positive solution for a class of nonlinear fractional differential equations. Acta Mathematica Scientia, 33(3), 847-854. 
[13] Wang, C., Gao, L., \& Dou, Q. (2014). Existence and uniqueness of positive solution for a nonlinear multi-order fractional differential equations. British Journal of Mathematics \& Computer Science, 4(15), 2137.

[14] Wang, X., Wang, L., \& Zeng, Q. (2015). Fractional differential equations with integral boundary conditions. Journal of Nonlinear Sciences and Applications, 8, 309-314.

[15] Wang, C., Zhang, H., \& Wang, S. (2012). Positive solution of a nonlinear fractional differential equation involving Caputo derivative. Discrete Dynamics in Nature and Society, 2012, Article ID 425408, 16 pages.

[16] Yan, R., Sun S., \& Sun, Y. (2016). Existence and multiplicity of solutions for fractional differential equations with parameters. Journal of Applied Mathematics and Computing, 51(1-2), 109-125.

[17] Zhang, S. (2000). The existence of a positive solution for a nonlinear fractional differential equation. Journal of Mathematical Analysis and Applications, 252(2), 804-812.

[18] Nanware, J.A., \& Dhaigude, D.B. (2014). Existence and uniqueness of solutions of differential equations of fractional order with integral boundary conditions. Journal of Nonlinear Sciences and Applications, 7(4), 246-254.

[19] V. Daftardar-Gejji, V., \& Jafari, H. (2007). Analysis of a system of nonautonomous fractional differential equations involving Caputo derivatives. Journal of Mathematical Analysis and Applications, 328, 026-1033.

[20] Zhou, Y. (2014). Basic theory of fractional differential equations, (Vol. 6). Singapore: World Scientific. 